\title{
Acoustic Absorption of Passive Destructive Interference Cavities
}

\author{
Arun Arjunan ${ }^{\mathrm{a} *}$ \\ aStructural Mechanics and Vibro-acoustics Research Group, School of Engineering, University \\ of Wolverhampton, Telford Innovation Campus, TF2 9NT, United Kingdom
}

\begin{abstract}
Acoustic products are primarily designed for broadband acoustic absorption. However, frequency-dependent acoustic absorption featuring passive designbased solutions are necessary to combat the growing noise pollution. Accordingly, this research investigates the targeted creation of sound absorption as a function of geometry utilising the principle of Acoustic Interference (AI). A methodology to design freeform geometries that can create targeted acoustic absorption is presented. The effectiveness of this methodology is then experimentally validated while quantifying the influence of length, diameter and geometry orientation. The results establish that AI has the potential to create 'near perfect' sound absorption that can be customised depending on the source frequency. The design freedom revealed by this study allows the exploitation of freeform geometries as passive highefficiency sound absorbing devices.
\end{abstract}

Keywords: Sound Absorption Coefficient; Polyamide-12; Additive Manufacturing; Acoustic Interference; Passive Noise Cancellation

\footnotetext{
* Corresponding author: SA230, Structural Mechanics and Vibro-acoustics Research Group, School of Engineering, University of Wolverhampton, Telford Innovation Campus, Telford, TF2 9NT, UK

Tel.: +44 1902 323829; fax: +44 01902323843

E-mail address: a.arjunan@wlv.ac.uk (Dr. Arun Arjunan)
} 


\section{Introduction}

Broadband acoustic absorbers are extensively used for noise control application. These acoustic absorbers can be broadly classified into porous and resonant [1-3] with the latter involving Helmholtz and quarter-wave resonators [4,5], perforated panels [6], and membrane absorbers [7]. Even though the narrow frequency band exhibited by these absorbers are undesirable, the high sound absorption coefficient $(\alpha)$ is suitable from a noise reduction perspective [8,9]. Accordingly, such sound absorbers are called 'bass traps' when used for low frequency attenuation [10].

Noise reduction technologies that can create 'tunable' [11] sound absorption is suitable for maximising noise reduction. While numerous approaches have already being sought in this regard $[12,13]$; noise pollution is still a challenging problem that affects around 4,860 homes in England [14]. This is because, the performance of traditional sound absorption technologies depend on mass, stiffness and surface properties [15-18] of the material leaving only limited possibilities for design-based interventions. However, for enhanced acoustic absorption, design-based strategies, as opposed to materials, are required [19]. Accordingly, this research explores the development of acoustic absorbers that utilises the principles of Acoustic Interference (AI) [20,21] where the sound absorption can be tailored based on the sound source frequency.

Geometry based interference cavities that can manipulate the phase difference of an acoustic wave can achieve superior noise cancellation [22]. However, like resonance-based technologies, interference absorbers are only effective at a narrow frequency range. However, the material independent performance of these absorbers allows for coupling with broadband technologies to improve the sound absorption substantially. This allows for targeted absorption of low-frequency noise that is often a challenge for traditional sound absorbers [23,24].

Attempts to create acoustic interference dates back to the works of Herschel [25] and Quincke [26] where, parallel connection of two pipes called HQ waveguides was experimented. However, the configuration received little attention in comparison to Helmholtz and quarter-wave resonators [27] due to its complex design. Furthermore, the practical application of these waveguides was limited to parallel pipes that can be interconnected using a side branch. Consequently, the utilisation of interference theory for passive sound absorption is only possible if interference can be created at the entrance of the waveguide and not 'inside'. Consequently, further research is necessary to develop geometries that can deliver interference within an alternative design. Accordingly, this research proposes and investigates free-form cavities that can create acoustic interference at the entrance. In addition, a one-dimensional relationship that links the cavity length to the frequency of $\alpha$ is also demonstrated.

Recent literature [28-30] depicts various interventions ranging from coatings to the use of metallic an hybrid foams to improve acoustic absorption. A design strategy to develop micro- 
perforated panel absorber was explored by Qian and Zhang [31]. While Jiang and Huang [32] characterised low-frequency acoustic wave propagation through a corrugated waveguide. Furthermore, the influence of tube bend on the performance of quarter-wave resonators was studied by Cambonie et al. [33]. Despite the substantial literature, design-based interventions suitable to reduce broadband noise is limited [34,35].

The exception to this is the pioneering works by Godbold, Soar, and Buswell [36], where interference cavities were benchmarked against conventional resonators showing that interference can take arbitrary and conformal paths. This was followed by Setaki et al. [21] who presented a potential application of interference on building acoustics. Further to this, the sound absorption of free-form geometries and two-dimensional planar cavities were studied by Berardi [37] and Cai et al. [38] respectively.

Outside of these studies, the literature on the sound absorption of interference cavities is scarce. This is due to the challenge in manufacturing complex geometrical cavities that can create AI within a 'usable volume'. However, the rise in 3D printing techniques [39-43] such as Selective Laser Sintering (SLS) is changing the way geometrically precise cavities can be fabricated $[44,45]$. Consequently, additive manufacturing has been finding its application on the development of customisable sound absorbing materials.

Acoustic properties of an additively manufactured porous polycarbonate were evaluated by Liu et al. [46]. Nansha and Hong [47] developed a micro-helix metamaterial by 3D printing and showed that helix vestibule and cavity depth had a significant impact on $\alpha$. Furthermore, design at the material microstructure level for low frequency sound attenuation featuring a periodic array of resonators were presented by Dupont et al. [48].

Contributing to this ambition of design-driven performance, this study investigates the feasibility of developing AI cavities that can be customised for the source frequency. A non-traditional approach incorporating all interference parameters into a single cavity is demonstrated for the first time. The manufacturing is realised by taking advantage of digital manufacturing technologies such as SLS. The emphasis of the study was to demonstrate the targeted sound absorption of three different frequencies using the principle of AI. Consequently, this paper aims to establish a new viewpoint on design-based noise reduction. Furthermore, the influence of cavity length, diameter, and orientation on $\alpha$ peaks are also investigated. The design methodology presented in this paper can accelerate the development of freeform geometries that can fully absorb sound at targeted frequencies. This can aid in the creation of innovative acoustic devices that are material independent, scalable and customisable. 


\section{Materials and Methods}

\subsection{The principle behind the proposed design-based interference}

The principle behind the proposed concept to create acoustic Destructive Interference (DI) is to use sound waves from a common source simultaneously entering through both ends of a geometrical cavity. The amount of phase shift between the waves depends on the relative length of the cavity and the wavelength of the sound wave. When the difference in wavelength is half the incident wavelength, a $180^{\circ}$ phase shift will occur cancelling the sound. Therefore, for a cavity length $l$ under constant sonic velocity $c$, the frequency of the sound wave and the interference length $(\delta l)$ can be related using the one-dimensional Eqn. (1):

$$
\delta l=l_{2}-l_{1}=\frac{(2 n-1) c}{2 f} \quad\{n=1,2,3 \ldots\}
$$

Where, $n$ is a positive integer and, $l_{1}$ and $l_{2}$ are long and short paths of the cavity. While passive interference is known to alter the acoustic performance, the challenge for this research is to investigate the possibility of designing an acoustic absorber that utilises interference for passive destruction and not in the commonly used active form. The benefit of this approach is the increased design flexibility that allows for free-form designs as compared to commonly used resonance approaches such as quarter-wave [50] or Helmholtz resonators [51]. As can be seen, the proposed system dismisses the requirement for acoustical signal monitoring or generation as featured in active systems.

\subsection{Selection of interference frequency and cavity design}

To create sound absorption at the cavity entrance; it is proposed that the length $l$ of the total cavity is equal to the interference length $\delta l$ as shown in Eqn. (2):

$$
\frac{l}{\delta l}=1
$$

At an interference ratio of 1 , three different frequencies $0.5,1$ and $1.5 \mathrm{kHz}$ are selected for the parametric study. Using these frequencies, the $\delta l$ was predicted to be $34.3,17.15$ and $11.43 \mathrm{~cm}$ at $0.5 \mathrm{kHz}, 1 \mathrm{kHz}$ and $1.5 \mathrm{kHz}$ respectively. For each $\delta l$, a total of four designs are considered at diameter $(d) 1.5 \mathrm{~cm}, 2 \mathrm{~cm}, 2.5 \mathrm{~cm}$ and $3 \mathrm{~cm}$. The limiting factor of the largest cavity diameter of $3 \mathrm{~cm}$ was the necessity to accommodate the longest $\delta l$ of $34.3 \mathrm{~cm}$ within a cylinder of diameter $(D)$ and height $(h) 10 \mathrm{~cm}$. The global diameter $(D)$ and height $(h)$ were prescribed by the dimensions of the experimental setup required to conduct the measurement of $\alpha$ between 0.4 and $1.6 \mathrm{kHz}$.

Even though a simple ' $U$ ' shaped cavity was the initial choice due to its scalable simplicity, alternative cavity shapes are considered to study the impact of shape orientation. In addition to $\mathrm{U}$, two spiral shapes namely $\mathrm{H}$ (spiral) and I (twin spiral) featuring similar interference length 
were designed. However, for the later, the diameter was limited to $2 \mathrm{~cm}$ due to the global dimensional constraints.

Table 1 Design parameters considered for the interference cavities.

\begin{tabular}{|c|c|c|c|c|c|}
\hline \multirow{2}{*}{ Type } & \multirow{2}{*}{$\begin{array}{c}\text { Cavity length }(\mathbf{c m}) \\
\delta l\end{array}$} & \multicolumn{4}{|c|}{ Cavity diameter $(\mathrm{cm})$} \\
\hline & & $d_{1}$ & $d_{2}$ & $d_{3}$ & $d_{4}$ \\
\hline $\mathrm{U}$ & $34.3(\mathrm{~A})$ & $3(3 \mathrm{AU})$ & $2.5(2.5 \mathrm{AU})$ & $2(2 \mathrm{AU})$ & $1.5(1.5 \mathrm{AU})$ \\
\hline $\mathrm{U}$ & $17.15(\mathrm{~B})$ & $3(3 \mathrm{BU})$ & $2.5(2.5 \mathrm{BU})$ & $2(2 \mathrm{BU})$ & $1.5(1.5 \mathrm{BU})$ \\
\hline $\mathrm{U}$ & $11.43(\mathrm{C})$ & $3(3 \mathrm{CU})$ & $2.5(2.5 \mathrm{CU})$ & $2(2 \mathrm{CU})$ & $1.5(1.5 \mathrm{CU})$ \\
\hline $\mathrm{H}$ & $34.3(\mathrm{~A})$ & - & - & $2(2 \mathrm{AH})$ & - \\
\hline I & $34.3(\mathrm{~A})$ & - & - & $2(2 \mathrm{AI})$ & - \\
\hline S & No cavity $(\mathrm{N})$ & $0(0 \mathrm{NS})$ & - & - & - \\
\hline
\end{tabular}

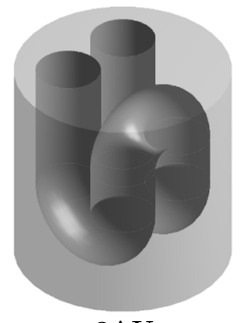

3AU

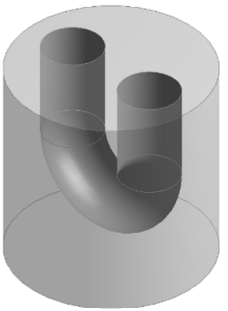

3BU

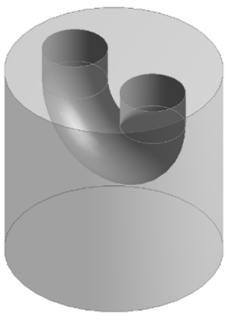

$3 \mathrm{CU}$

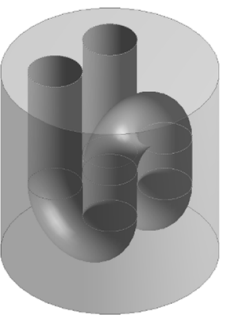

$2.5 \mathrm{AU}$

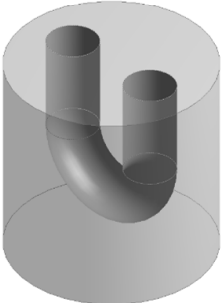

2.5BU

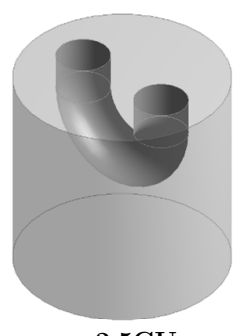

$2.5 \mathrm{CU}$

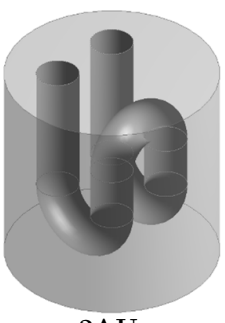

$2 \mathrm{AU}$

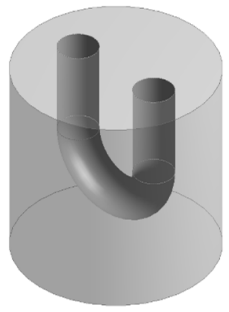

2BU

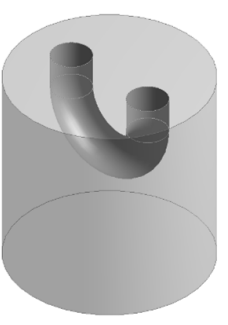

2CU

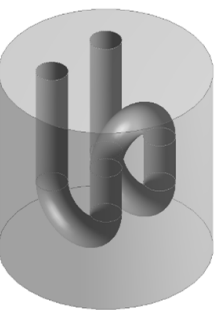

$1.5 \mathrm{AU}$

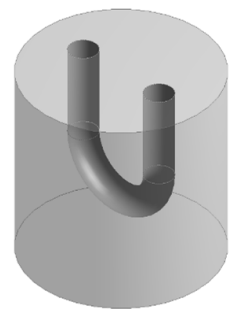

1.5BU

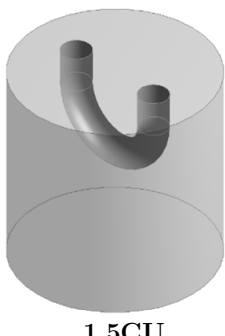

$1.5 \mathrm{CU}$

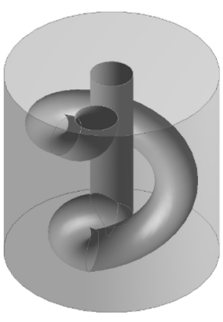

$2 \mathrm{AH}$

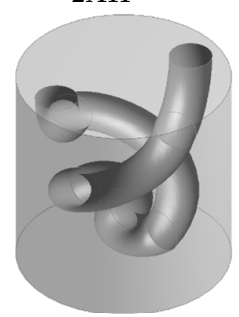

2AI

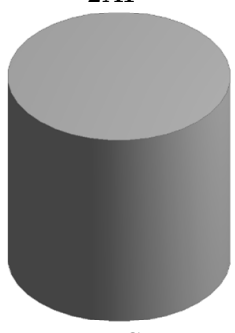

0NS

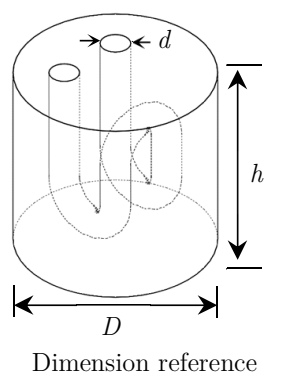

Fig. 1. Interference cavity designs considered. 
While the objective of shape selection was primary acoustic, consideration was also given for easy removal of the Polyamide-12 (PA12) powder resulted from the SLS process[52]. Furthermore, a solid specimen design with no cavities was considered to characterise surface absorption coefficient of PA12. Altogether, a total of 15 different designs were generated with associated parametric values listed in Table 1 . The designs were digitally produced using the SolidWorks (Dassault Systèmes) design package, the results of which are shown in Fig. 1.

\subsection{Manufacturing}

The designs were Additively Manufactured (AM) using the SLS process in PA12 offered by '3D Systems' with an average grain size of $56 \mu \mathrm{m}$. The laser sintering of PA12 is a manufacturing process where a solid body is created out of an unstructured and formless powder bed using focused laser power. This means that besides the shape, the properties of the part will also be created to a certain extent during the manufacturing process [53]. Accordingly, the properties of the processed material are as listed in Table 2. PA12 was the material of choice due to the relatively low sound absorption it inherently possesses within the frequency range under consideration. This ensured that the sound absorption is a function of geometry with minimal influence from the material itself.

Table 2. Material properties of Polyamide-12 powder used for sintering.

\begin{tabular}{ll}
\hline Properties & Values \\
\hline Density & 0.9 to $0.95 \mathrm{~g} / \mathrm{cm}^{3}$ \\
Young's Modulus & $1700 \pm 150 \mathrm{MPa}$ \\
Tensile Strength & $45 \pm 3 \mathrm{MPa}$ \\
Elongation at Break & $20 \pm 5 \%$ \\
Melting Point & 172 to $180^{\circ} \mathrm{C}$ \\
Thermal Expansion & $1.09 \times 10^{-4} / \mathrm{K}$ \\
\hline
\end{tabular}

All the 15 designs were exported into the Standard Tessellation Language (STL) for preprocessing. The files were then imported into Materialise Magics to generate layer data required for the manufacturing process. The parts were oriented on the platform in such a manner as to reduce overhang during the printing process. This method allowed the parts to be self-supported without the need of any support structures resulting in a part that required minimal postprocessing. This was crucial to obtain a smooth cavity surface, as remnants of support-structures could interfere with the transmission of the acoustic wave through the interference cavities.

'Sinterstation-2500' developed by '3D Systems' was used for the sintering process. On receiving the sliced STL file, the machine warms up with PA12 powder heated to just below the melting point. At a scan speed of $10 \mathrm{~m} / \mathrm{s}$, the first layer is then traced out by a CO2 laser that melts and fuses the material upon contact. The most important factor that influences the quality of 
the sintering process is the energy density $\left(E_{d}\right)$. The process parameters such as laser power $\left(P_{l}\right)$, scan spacing $\left(S_{s}\right)$ and laser beam speed $\left(v_{b}\right)$ were then related using Eqn. (3):

$$
E_{d}=\frac{P_{l}}{v_{b} \times S_{s}}
$$

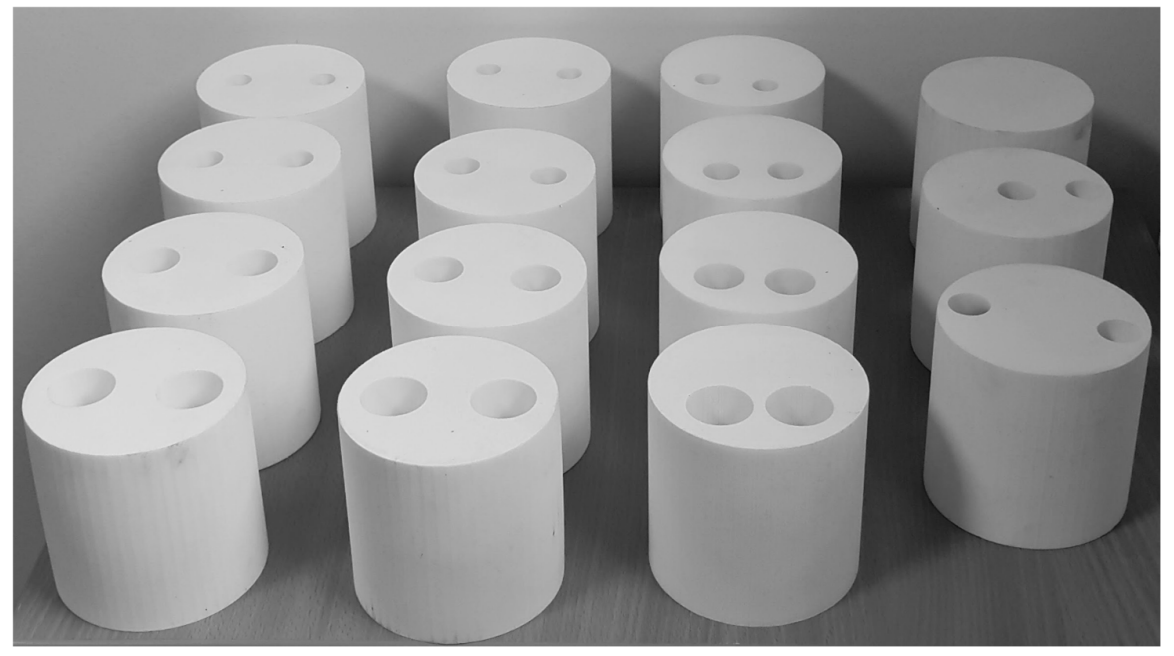

Fig. 2. Additively manufactured PA12 test specimens.

Other processes like the part bed temperature were adjusted and optimised so that a process control part could be produced with maximum resolution at $0.1 \mathrm{~mm}$ layers. Once the sintering process completed, the parts were allowed to stand and cool down before being removed from the build platform. The un-sintered powder from the geometrical cavities was then removed using compressed air. The end-product of the 15 test specimens additively manufactured are shown in Fig. 2.

\subsection{Experimental measurement}

To assess acoustic performance, $\alpha$ was characterised using an impedance tube-based transferfunction complying with ISO10534-2 [54]. Measurements were taken with the impedance tube in the horizontal position at a relative humidity of $64 \%$ and temperature of $20{ }^{\circ} \mathrm{C}$. An in-tube loudspeaker connected to a function generator via a PA50 power amplifier and a Fast Fourier Transform (FFT) spectrum analyser was used as the acoustic exciter. The sound pressure signals from the impedance tube were monitored in terms of amplitude and recorded by the two microphones $m_{1}$ and $m_{2}$. Microphone $m_{1}$ was the closest to the test sample at $15 \mathrm{~cm}$ and $m_{2}$ was at $8 \mathrm{~cm}$ from $m_{1}$ towards the sound source. Both the microphones and the PA50 were connected to the data acquisition hardware and a workstation running VA-Lab. 
From the acoustic amplitudes $p_{1}$ and $p_{2}$ measured by microphones $m_{1}$ and $m_{2}$ respectively, the transfer function $H_{12}$ and normal incident sound reflection factor $r$ was computed using Eqn. (4) and (5). Where, $P_{1}$ and $P_{2}$ are the signals transformed from $p_{1}$ and $p_{2}, r_{r}$ and $r_{i}$ denote the 'real' and 'imaginary' components of the reflected sound, $s$ is the distance between the two microphones and $x_{1}$ is the linear distance from the specimen surface to the furthest microphone. Furthermore, $k=2 \pi f / c$, the wave number was calculated as a function of the sonic velocity $c$ and the incident sound frequency $f$. Accordingly, the sound absorption coefficient characterising the acoustic performance of the interference cavities were calculated using Eqn. (6):

$$
\begin{gathered}
H_{12}=\frac{P_{2}}{P_{1}} \\
r=r_{r}+j r_{i}=\frac{H_{12}-e^{-j k s}}{e^{j k s}-H_{12}} e^{2 j k x_{1}} \\
\alpha=1-|r|^{2}
\end{gathered}
$$

The impedance tube diameter of $10 \mathrm{~cm}$ was dictated by the frequency range considered (0.4-1.6 $\mathrm{kHz}$ ) for the measurements. The microphones were calibrated and preliminary tests using a known sample showed that the experimental setup was reliable and repeatable. The signal amplitude at the microphone location was at least $10 \mathrm{~dB}$ higher than the background noise at all frequencies tested. Using the two-microphone setup, correction of the measured transfer function data for channels mismatch was done through repeated measurements with microphone channels interchanged.

Consequently, for each design, six measurements were taken at one-twelfth octave band range, three measurements each with the microphones interchanged. The standard practice in acoustics is to use octave bands when it comes to the measurement of sound absorption coefficient. Either a one-third or one-twelfth octave band is recommended as best practice depending on the resolution of data required.

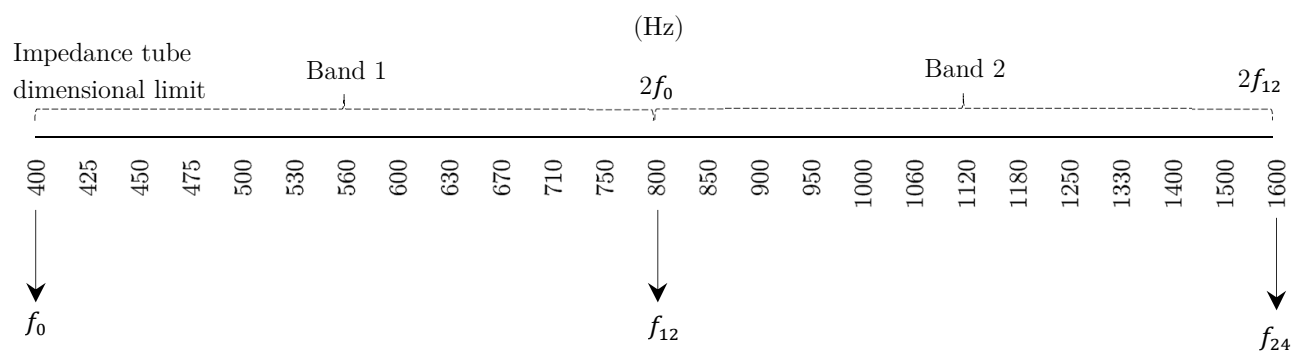

Fig. 3. The frequency band used for the measurement of sound absorption coefficient.

In this study, a one-twelfth octave band is used to obtain the highest resolution of data possible. Using this technique, the frequency range is represented as the width of an octave where the 
upper band frequency is twice the lower band frequency, where the step size is represented by Eqn. (7):

$$
\frac{f_{n+1}}{f_{n}}=2^{k}
$$

Where, $k=1 / 12$. This will give rise to two bands with frequency as shown in Fig. 3 . The frequency scale is also published in ISO10140-1:2016 [55]. The measurements were then used to obtain the final frequency dependent $\alpha$ of the interference cavities and the solid specimen.

\section{Results and discussion}

\subsection{Validation of the test setup}

A Polyurethane foam of known $\alpha$ was used as a measurement standard. The standard had a density $6 \mathrm{~kg} / \mathrm{m}^{3}$, diameter $10 \mathrm{~cm}$ and thickness $2.5 \mathrm{~cm}$. Fig. 4 shows the measured sound absorption with respect to reference data from independent tests at one-third octave band. On comparison, the maximum difference between the data sets was $6 \%(0.06)$, which is well within the acceptable uncertainty of $10 \%$ (0.1) [56]. A $10 \%$ variation in $\alpha$ only translates to an uncertainty of $\sim 0.5 \mathrm{~dB}$ in the sound power absorbed; an acceptable case as discussed by Han et al. [56]. Therefore, the presented two-microphone experimental rig follows relevant standards.

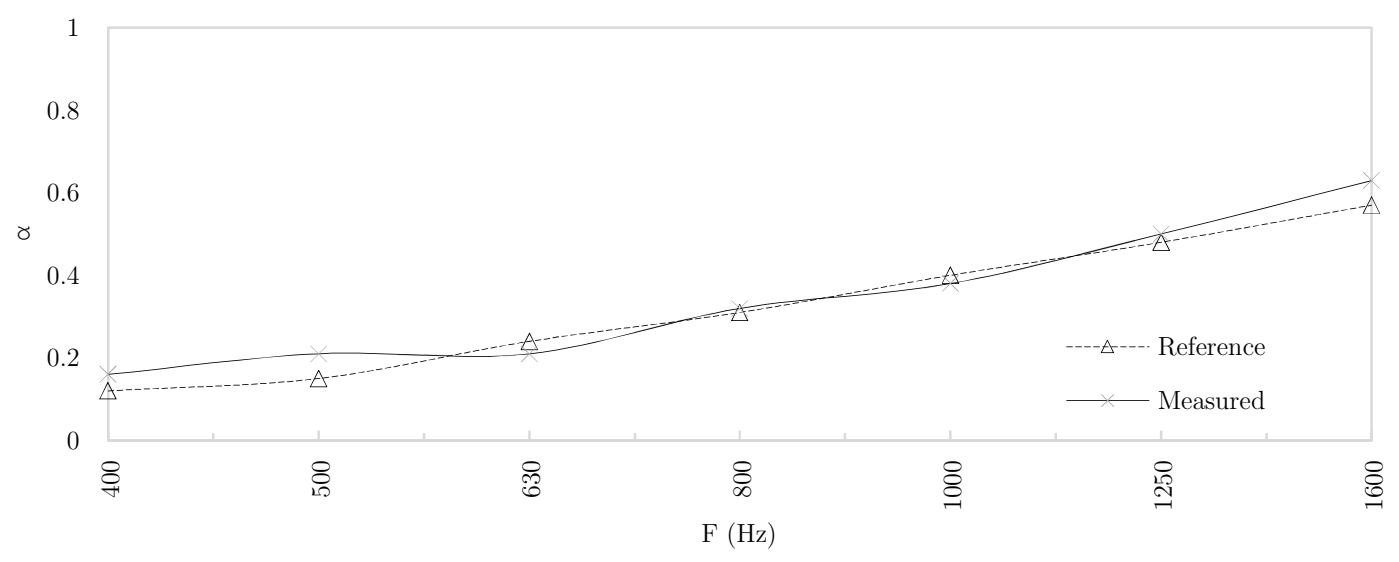

Fig. 4. Reference and measured $\alpha$ for test setup validation at one-third octave bands.

\subsection{Targeted creation of design-based interference}

The fundamental research question is whether the modified interference theory can create destructive interference in the absence of a traditional side branch. For a $\delta l / l$ ratio of 1 , an interference length of $17.15 \mathrm{~cm}$ had a theoretical frequency of $500 \mathrm{~Hz}$ at $\mathrm{n}=1$ and $1500 \mathrm{~Hz}$ at $\mathrm{n}=2$ where destructive interference can occur. As shown in Fig. 5, noise cancellation occurred at $475 \mathrm{~Hz}$ and $1400 \mathrm{~Hz}$ exhibiting sound absorption values of 0.96 and 0.98 at $\mathrm{n}=1$ and 2 respectively. However, a slight shift between the theoretical and the experimental frequency of $5 \%$ and $7 \%$ was observed for $\mathrm{n}=1$ and 2 respectively. 


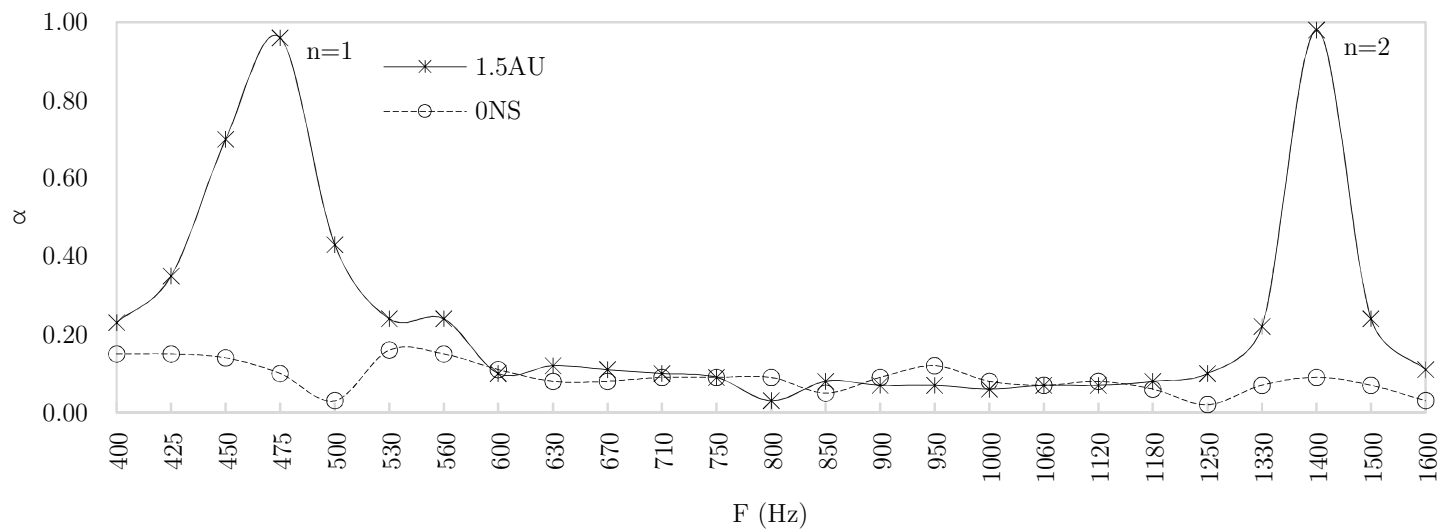

Fig. 5. Measured $\boldsymbol{\alpha}$ for designs 1.5AU and 0NS.

As listed in Table 3 , for all $\delta l$, this shift in frequency between the theoretical and experimental was observed. However, for a given design frequency, this shift was not influenced by cavity diameter or orientation. Consequently, the experimental frequency shift can be associated to the absence of a side branch.

Table 3. Comparison between theoretical $\left(\boldsymbol{F}_{\boldsymbol{t h} \boldsymbol{r}}\right)$ and experimental $\left(\boldsymbol{F}_{\text {exp }}\right)$ frequencies observed.

\begin{tabular}{cccccc}
\hline Design & $\boldsymbol{\delta} \boldsymbol{l}(\mathrm{cm})$ & $\mathbf{n}$ & $\boldsymbol{F}_{\boldsymbol{t h \boldsymbol { r }}}(\mathrm{Hz})$ & $\boldsymbol{F}_{\boldsymbol{e x} \boldsymbol{p}}(\mathrm{Hz})$ & $\%$ difference \\
\hline 3AU, 2.5AU, 2AU, 1.5AU, & 34.3 & 1 & 500 & 475 & 5 \\
2AH and 2AI & 34.3 & 2 & 1500 & 1400 & 7 \\
3BU, 2.5BU, 2BU and 1.5BU & 17.15 & 1 & 1000 & 950 & 5 \\
3CU, 2.5CU, 2CU and 1.5CU & 11.43 & 1 & 1500 & 1330 & 12 \\
\hline
\end{tabular}

Before fully associating the absorption peaks with interference, it was important to rule out any contribution from eigenmodes of the impedance tube itself. Accordingly, a solid PA12 prototype with similar global dimensions 0NS was tested and compared as shown in Fig. 5. Analysing the results, it is evident that the absorption peaks are a distinct contribution of the interference cavities. The solid specimen 0NS with no cavities not only failed to recreate any interference peaks but also exhibited poor absorption at all frequencies similar to what can be expected from a fully dense PA12 surface.

Other than for the selected frequencies both $0 \mathrm{NS}$ and 1.5AU performed similarly further demonstrating the performance of the interference cavities. Consequently, the results validate noise cancellation through geometry-based interference where the resulted peaks $475 \mathrm{~Hz}$ and $1400 \mathrm{~Hz}$ can be associated with the first and second mode at $\mathrm{n}=1$ and $\mathrm{n}=2$ respectively. Furthermore, $\alpha$ values close to 1 (0.96 and 0.98) clearly show the potential for high performance low frequency dependent sound absorbing devices, which has always been a challenge from a noise control perspective. 
In most cases, alternative design-based treatment such as expansion chamber, quarter-wave tube, or the Helmholtz resonator is only effective for transmission or insertion loss [57] and not for sound absorption. This further demonstrates the significance of the demonstrated interference cavities for design-based sound absorption. Analysing the width of the absorption peaks as referred by Godbold et al. [36] a maximum average of 100 Hz can be associated, which further shows the potential of the interference cavities. For 1.5AU, the peak width was found to be constant at $n=1$ and $n=2$.

\subsubsection{Influence of cavity diameter}

For an interference length of $34.3 \mathrm{~cm}$, all the four designs exhibited absorption peaks associated with the design frequency as shown in Fig. 6. However, with the increase in diameter the peak $\alpha$ as a result of DI reduced steadily. The highest $\alpha$ was exhibited by the smallest cavity diameter at both $\mathrm{n}=1$ and 2 . Even though all the designs under this category exhibited DI based absorption, a variation in amplitude was observed at $n=2$; where the highest $d$ resulted in the lowest $\alpha$ (0.38); reducing the diameter increased $\alpha$ with $1.5 \mathrm{~cm}$ resulting in almost complete absorption of 0.98 .

Lower sound absorption at a larger diameter can be due to the three-dimensional interference facilitated by the larger cavity volume. Consequently, the significance of this effect can be expected to increase with an increase in frequency. However, this aspect of the cavity requires further study and an improvisation of the one-dimensional theory is required. However, in comparison to the solid specimen, all interference designs exhibited superior sound absorption.

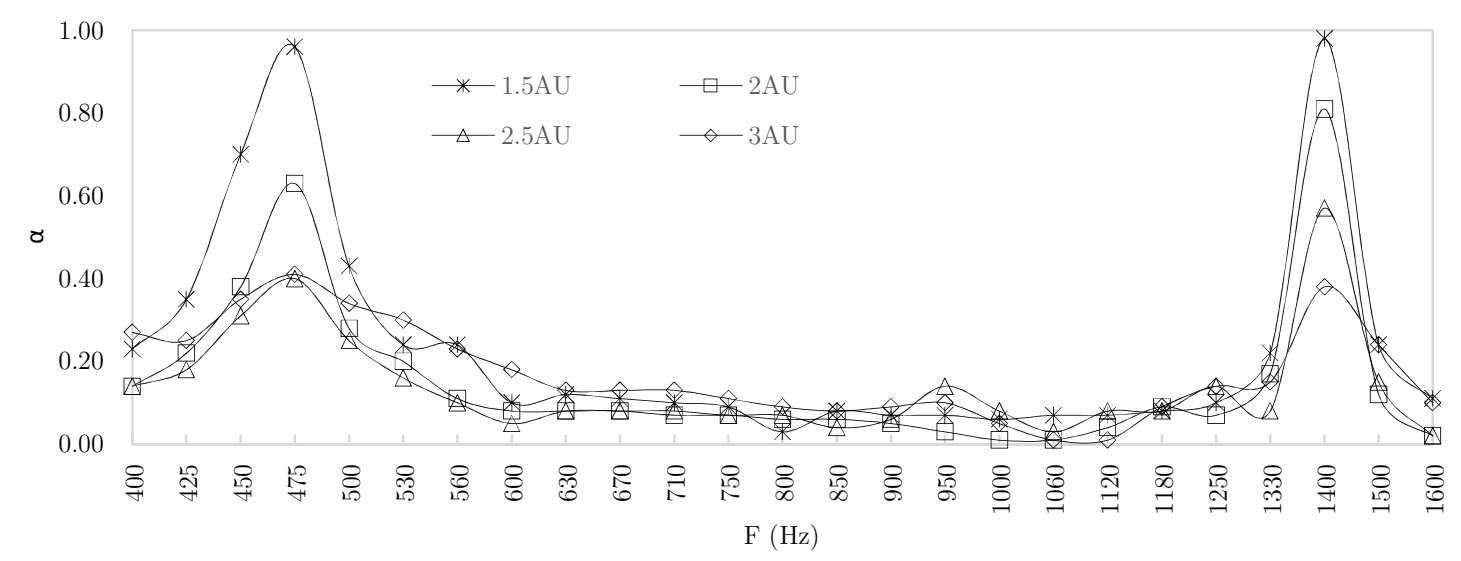

Fig. 6. Influence of diameter on $\boldsymbol{\alpha}$ of type U designs featuring an interference length of $34.3 \mathrm{~cm}(\mathrm{~A})$.

At $\mathrm{n}=1$, DI exhibited lower $\alpha$ in comparison to the second peak at $\mathrm{n}=2$. While diameters $1.5,2$ and $2.5 \mathrm{~cm}$ showed comparable performance to the second peak the $\alpha$ values where comparatively lower. However, diameter $3 \mathrm{~cm}$ and $2.5 \mathrm{~cm}$ at $\mathrm{n}=1$ exhibited similar performance with a broader half-peak width performance. The lower peak absorption can be the result of the compound interference due to the lower $\delta l / d$ ratio of the cavity design. 
The geometries tested under the category $\mathrm{BU}(\delta l=17.15 \mathrm{~cm})$ also showed $\alpha$ peaks representative of destructive interference as shown in Fig. 7. The peak absorption for all diameters in this category were observed around $950 \mathrm{~Hz}$ in comparison to the theoretical frequency of $1000 \mathrm{~Hz}$. Nevertheless, the difference between the theoretical and experimental frequency was consistent with previous results.

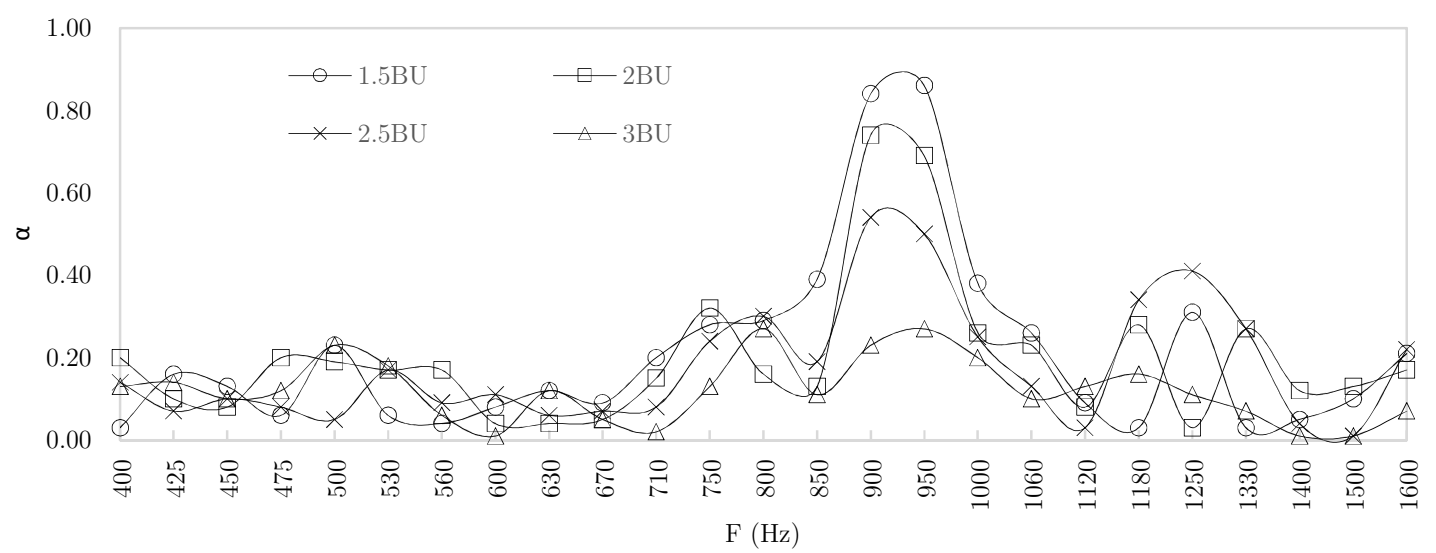

Fig. 7. Influence of diameter on $\boldsymbol{\alpha}$ of type U designs with an interference length of $17.15 \mathrm{~cm}$ (B).

Furthermore, the sound absorption at peak DI varied significantly depending on the design diameter. Peak $\alpha$ for each design was observed to be $0.86,0.69,0.50$ and 0.27 for diameters 1.5, $2,2.5$ and $3 \mathrm{~cm}$ respectively resulting in the best performance at the lowest diameter and worst at highest. However, the change in peak amplitude of absorption was consistent with each 0.5 $\mathrm{cm}$ increase in cavity diameter. Different to previous designs, noticeable absorption was observed around $1250 \mathrm{~Hz}$, this could be associated with the resonance of expansion chambers. From an application perspective, these additional peaks improve the overall acoustic performance of the absorbers. Nevertheless, understanding of the related resonance modes needs continued analysis.

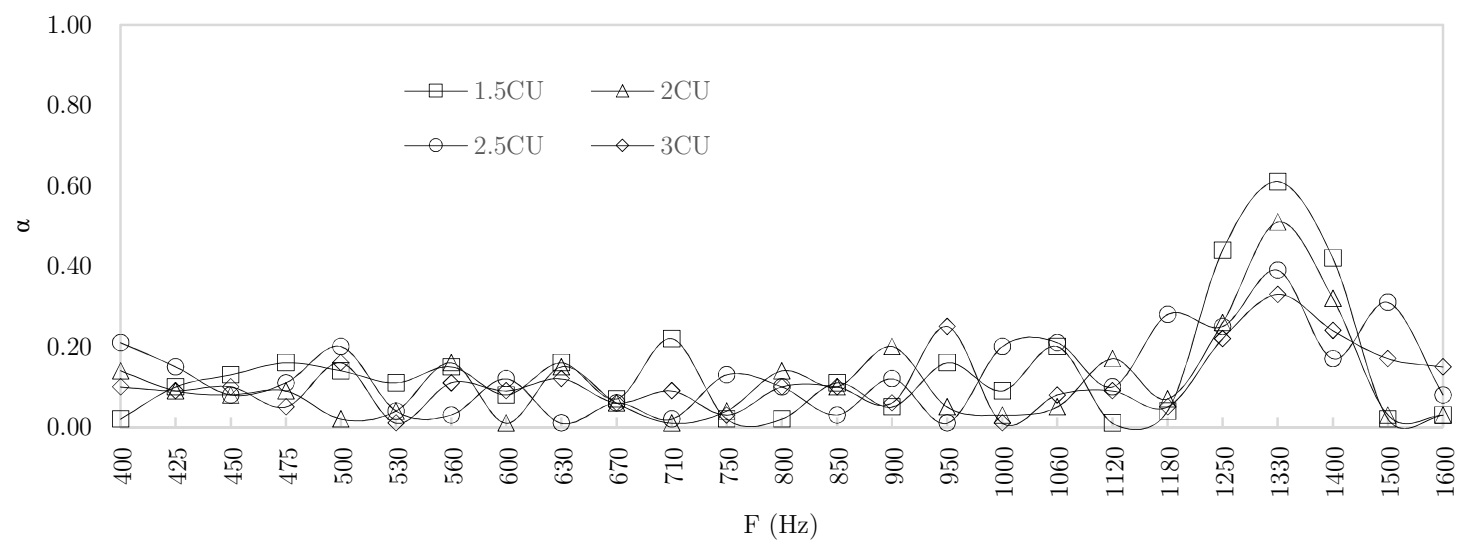

Fig. 8. Influence of diameter on $\alpha$ of type $U$ designs with an interference length of $11.43 \mathrm{~cm}(\mathrm{C})$.

The $\alpha$ for designs with the smallest interference length are presented in Fig. 8. The results show that the interference pattern is consistent with the observations so far. The absorption peaks 
were observed at $1330 \mathrm{~Hz}$ for all the four diameters tested under this category, a slight shift from the theoretical $1500 \mathrm{~Hz}$. The smallest and largest diameter exhibited the highest and lowest $\alpha$ of 0.61 and 0.33 respectively.

When the interference frequency increased, larger diameters are exhibiting multiple resonances within a single geometry exhibiting many low-level absorption peaks. While these peaks can be clearly distinguished due to their comparatively low $\alpha$, this is an indication that at higher frequencies the cavity diameter plays an even greater role in obtaining a predictable acoustic performance. However, this is advantageous in improving the overall absorption performance in comparison to the solid specimen.

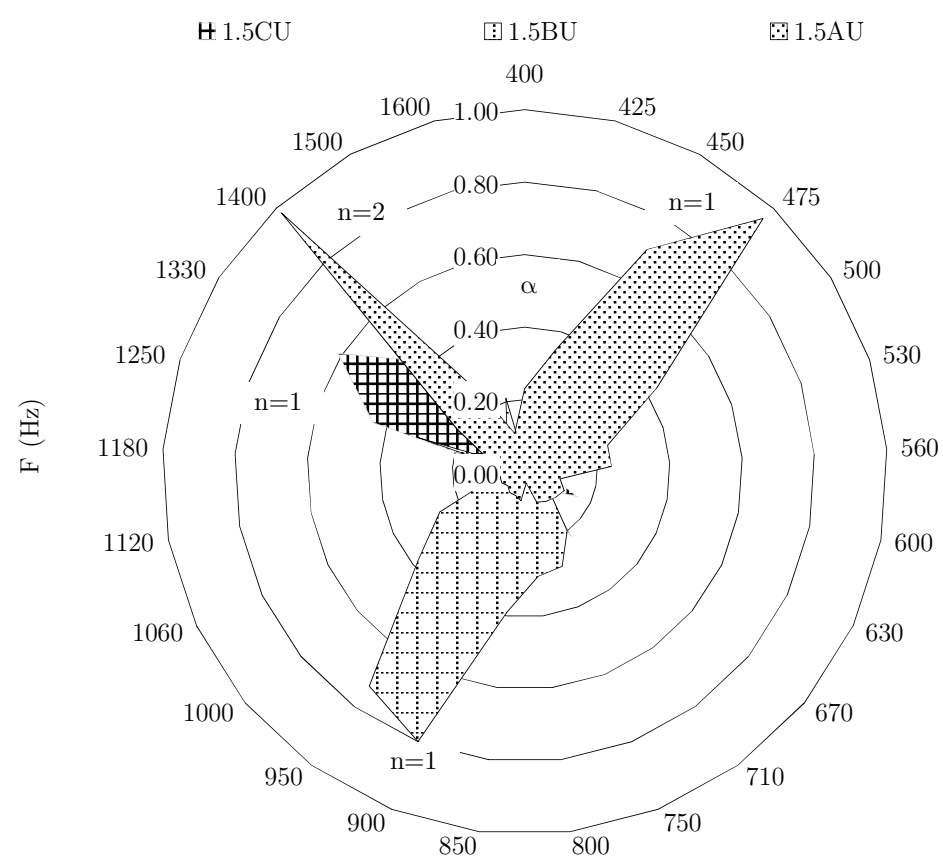

Fig. 9. Destructive interference-based sound absorption associated with best performing designs.

Fig. 9 shows the amplitude of peak $\alpha$ by the best performing designs under all the interference length under consideration. It is clear that for all frequencies, the smallest diameter exhibited the highest sound absorption. An $\alpha$ close to unity was exhibited by the longest interference length at $475 \mathrm{~Hz}(\mathrm{n}=1)$ and $1400 \mathrm{~Hz}(\mathrm{n}=2)$. However, such high $\alpha$ was not observed neither 900 nor $1330 \mathrm{~Hz}$ demonstrating the influence of $\delta l / d$ ratio. Nevertheless, a consistent reduction in $\alpha$ can be seen as the design frequency increases.

When $\delta l$ was reduced from 34.3 to $17.15 \mathrm{~cm}$, sound absorption reduced by $20 \%$. A similar pattern was observed on further reduction of $\delta l$ to $11.43 \mathrm{~cm}$. When $\mathrm{F}$ goes up the wavelengths reduces allowing for multiple interference through the cavity. This shows that further reduction in diameter are required at higher frequencies to reach peak sound absorption. Consequently, 
further parametric optimisation is required to find optimal cavity dimensions at higher frequencies. Looking at the half-peak width performance, performance at $n=1$ is comparable for all the cavity designs considered.

\subsubsection{Influence of geometry orientation}

The geometry orientation refers to the distribution of the cavity length along the primary interference direction. To characterise the influence of geometry orientation, designs AH and AI was developed. Despite designs 2AU, 2AH and 2AI featuring the same $\delta l$ and $d$ of 34.3 and $2 \mathrm{~cm}$ respectively, geometry orientation for all the three cavities are different. While AU follows a simple $\mathrm{U}$ shape, $\mathrm{AH}$ and $\mathrm{AI}$ are more complex following single and double helix patterns respectively. This helix was chosen to allow to utilise the maximum volume within the cylindrical specimen.

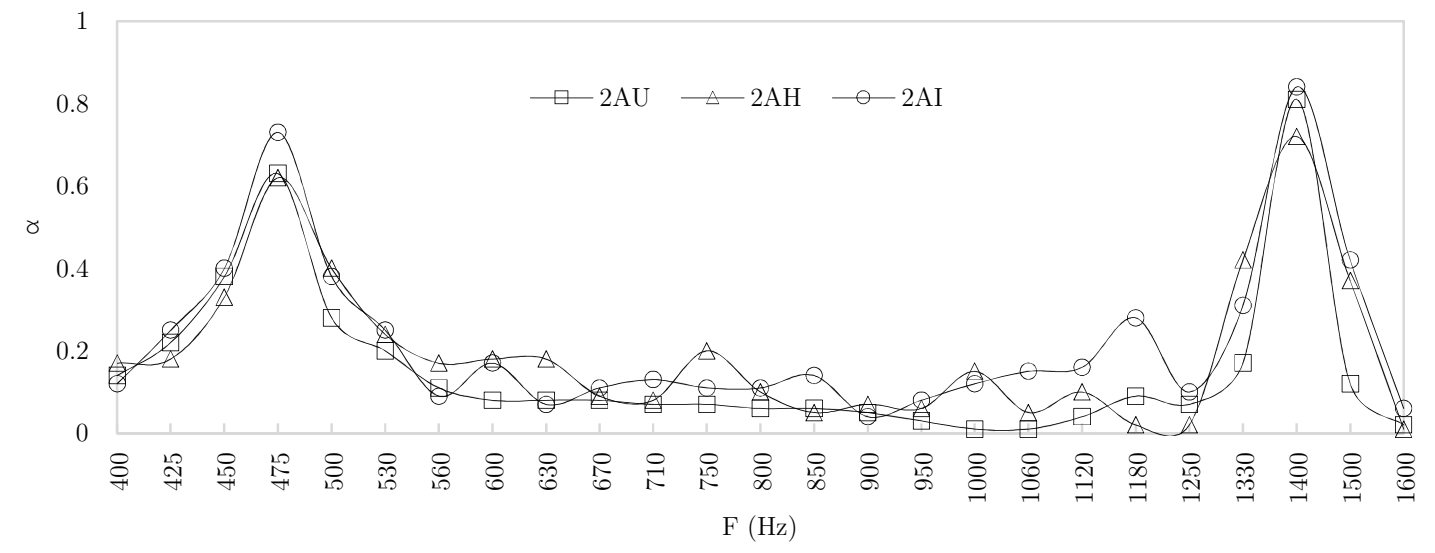

Fig. 10. Influence of geometry orientation on $\boldsymbol{\alpha}$ of interference cavities.

All the three designs exhibited DI at the predicted frequency as shown in Fig. 10. However, a slight shift in peak $\alpha$ values were observed between the cavities. 2AI exhibited the highest $\alpha$ of 0.73 at $\mathrm{n}=1$; a difference of 0.11 in comparison to the other cavities tested. However, at $\mathrm{n}=2$, both $2 \mathrm{AU}$ and $2 \mathrm{AI}$ exhibited similar sound absorption coefficients.

The results show that the change in geometry orientation leads to similar DI performance, however with a shift in $\alpha$ of approximately $16.3 \%$. The reason for this can be attributed to the variation in bend curvature; an effect observed in curved quarter-wave resonators [33]. Overall, 2AI showed superior sound absorption at the first interference peak. This makes it possible to accommodate larger lengths in smaller spaces from a design perspective. Nevertheless, this aspect of cavity design requires further investigation to come up with an optimum cavity shape.

Overall, the results of this study establish that interference cavities can be used to develop highefficiency design-based sound absorbing structures. The study also validated the performance of interference cavities in the absence of a side branch and set it apart from HQ waveguide. 
Furthermore, the sound absorption that can be expected by employing interference cavities at low frequencies is demonstrated. In comparison to solid specimen, interference cavities showed substantial improvement in $\alpha$ utilising the principle to destructive interference. These associations are critical in acting as design guidelines for further studies and parametric optimisation of interference cavities. It is anticipated that the results presented in this study can aid in the development of customisable noise abatement devices with superior sound absorption. Application include a multitude of areas; passive noise cancellation in small devices such as vacuum cleaners and lawn mowers to large scale sound absorption in motorways, railway lines and airports.

\section{Conclusions}

Strategies to conceive and manufacture interference cavities so that sound absorption occurs at the entrance of the cavity in the absence of a side branch is presented in this paper. The phenomenon of interference-based noise cancellation was experimentally investigated using 15 different PA12 prototypes. All the cavities exhibited interference peaks resulting in substantial improvement of $\alpha$ in comparison to a solid specimen of the same global dimensions and material. However, a slight shift in targeted frequencies was observed between theoretical prediction and experimental results due to the complex nature of the cavities and the absence of a side branch. The smallest diameter exhibited the highest absorption for all the interference cavities tested. Furthermore, a near perfect absorption of $0.96(n=1)$ and $0.98(n=2)$ was observed for the longest

interference length demonstrating near perfect noise cancellation ( $\alpha=1$ indicates $100 \%$ absorption). It was also found that the geometrical orientation of the cavities had a minimal influence on $\alpha$, allowing to develop free form geometries to fit any given space. The results presented in this study is expected to support the design of frequency dependent noise abatement devices exploiting the principles of acoustic interference. However, understanding the transmission loss of such cavities is also necessary to employ this technology for effective noise reduction.

\section{Acknowledgment}

This research was funded by the UK Department for Transport (DfT) under the Transport Technology Research Innovation Grant number T-TRIG150902 for primary research into highefficiency sound attenuating technologies for the UK High-Speed Rail (HS2) network.

\section{Data availability}

The data that support the findings of this study are available from the corresponding author upon reasonable request. 


\section{References}

[1] Cao L, Fu Q, Si Y, Ding B, Yu J. Porous materials for sound absorption. Composites Communications 2018;10:25-35.

[2] Zhao X-, Yu Y-, Wu Y-. Improving low-frequency sound absorption of micro-perforated panel absorbers by using mechanical impedance plate combined with Helmholtz resonators. Appl Acoust 2016;114:92-8.

[3] Zhai W, Yu X, Song X, Ang LYL, Cui F, Lee HP et al. Microstructure-based experimental and numerical investigations on the sound absorption property of open-cell metallic foams manufactured by a template replication technique. Mater Des 2018;137:108-16.

[4] CAI C, MAK CM. Noise attenuation capacity of a Helmholtz resonator. Adv Eng Software 2018;116:60-6.

[5] Howard CQ, Craig RA. Noise reduction using a quarter wave tube with different orifice geometries. Applied Acoustics 2014;76:180-6.

[6] Liu Z, Zhan J, Fard M, Davy JL. Acoustic properties of multilayer sound absorbers with a 3D printed micro-perforated panel. Applied Acoustics 2017;121:25-32.

[7] Gao N, Wu JH, Hou H, Yu L. Excellent low-frequency sound absorption of radial membrane acoustic metamaterial. Int J Mod Phys B 2017;31.

[8] Echevarria Sanchez GM, Van Renterghem T, Thomas P, Botteldooren D. The effect of street canyon design on traffic noise exposure along roads. Building and Environment 2016;97:96-110.

[9] Arjunan A, Wang CJ, Yahiaoui K, Mynors DJ, Morgan T, English M. Finite element acoustic analysis of a steel stud based double-leaf wall. Build Environ 2013;67:202-10.

[10] Kim S, Kim Y, Jang J. A theoretical model to predict the low-frequency sound absorption of a Helmholtz resonator array. J Acoust Soc Am 2006;119:1933-6.

[11] Cai X, Zhang Y, Yang J. Tunable ultra low and broad acoustic absorption by controllable pyrolysis of fiber materials. Materials Today Communications 2018;16:226-31.

[12] Arjunan A, Foteinou A. A comparative study on the acoustic behaviour of free-standing curved and flat single panel screens in an open-plan enclosed environment. INTER-NOISE - Int Congr Expo Noise Control Eng : Taming Noise Mov Quiet 2017;2017-January.

[13] Arjunan A. Sound Transmission Loss of Light-Weight Slotted Steel Studs in a Gypsum Plasterboard Partition Wall. INTER-NOISE 2016 (ISSN 0105-175x) 2016.

[14] Noise Action Plan: Railways (Including Major Railways) Environmental Noise (England) Regulations 2006, as amended. 2014;14126.

[15] Arjunan A, Wang C, Yahiaoui K, Mynors M, Morgan T, Nguyen B et al. Sound frequency dependent mesh modelling to simulate the acoustic insulation of stud based double-leaf walls. Proceedings of the 2014 Leuven Conference on Noise and Vibration Engineering (ISMA2014). 2014. [16] Arjunan A, Wang C, Yahiaoui K, English M. Finite Element Vibro-acoustic Simulation of Rollformed Steel Studs in Partition Walls. 2015.

[17] Arjunan A, Wang CJ, Yahiaoui K, Mynors DJ, Morgan T, Nguyen VB et al. Development of a 3D finite element acoustic model to predict the sound reduction index of stud based double-leaf walls. J Sound Vibrat 2014;333:6140-55.

[18] Arjunan A, Wang C, English M, Stanford M, Lister P. A Computationally-Efficient Numerical Model to Characterize the Noise Behavior of Metal-Framed Walls. Metals 2015;5:1414.

[19] Fang Y, Zhang X, Zhou J. Acoustic porous metasurface for excellent sound absorption based on wave manipulation. Journal of Sound and Vibration 2018;434:273-83.

[20] Arjunan A, Rackley J, Stanford M. Experimental investigation on the sound reduction performance of frequency controlled acoustic interference cavities. Proc INTER-NOISE - Int Congr Expo Noise Control Eng : Towar Quieter Future 2016:4211-9. 
[21] Setaki F, Tenpierik M, Turrin M, van Timmeren A. Acoustic absorbers by additive manufacturing. Building and Environment 2014;72:188-200.

[22] Elliott SJ, Nelson PA. Active noise control. IEEE Signal Process Mag 1993;10:12-35.

[23] Hu S, Rajamani R, Yu X. Directional cancellation of acoustic noise for home window applications. Applied Acoustics 2013;74:467-77.

[24] Sas P, Bao C, Augusztinovicz F, Desmet W. Active control of sound transmission through a double panel partition. Journal of Sound and Vibration 1995;180:609-25.

[25] Herschel JFW. On the absorption of light by coloured media, viewed in connexion with the undulatory theory. Philos.Mag. 1833;3:401-12.

[26] Quincke G. Interference apparatus for sound waves (English translation). Ann. Phys. Chem. 1866;128:177-92.

[27] Selamet A, Dickey NS, Novak JM. The Herschel-Quincke tube: A theoretical, computational, and experimental investigation. J Acoust Soc Am 1994;96:3177-85.

[28] Sharma GS, Skvortsov A, MacGillivray I, Kessissoglou N. Sound absorption by rubber coatings with periodic voids and hard inclusions. Applied Acoustics 2019;143:200-10.

[29] Otaru AJ. Enhancing the sound absorption performance of porous metals using tomography images. Applied Acoustics 2019;143:183-9.

[30] Park J, Yang SH, Minn KS, Yu CB, Pak SY, Song YS et al. Design and numerical analysis of syntactic hybrid foam for superior sound absorption. Mater Des 2018;142:212-20.

[31] Qian YJ, Zhang J. Engineering-oriented design strategy to obtain linear micro-perforated panel absorbers at high sound pressure environment. Applied Acoustics 2018;137:40-4.

[32] Jiang C, Huang L. Characterization of low-frequency acoustic wave propagation through a periodic corrugated waveguide. Journal of Sound and Vibration 2018;418:79-99.

[33] Cambonie T, Mbailassem F, Gourdon E. Bending a quarter wavelength resonator : Curvature effects on sound absorption properties. Applied Acoustics 2018;131:87-102.

[34] Field CD, Fricke FR. Theory and applications of quarter-wave resonators: A prelude to their use for attenuating noise entering buildings through ventilation openings. Applied Acoustics 1998;53:117-32.

[35] De Salis MHF, Oldham DJ, Sharples S. Noise control strategies for naturally ventilated buildings.

Building and Environment 2002;37:471-84.

[36] Godbold OB, Soar RC, Buswell RA. Implications of solid freeform fabrication on acoustic absorbers. Rapid Prototyping Journal 2007;13:298-303.

[37] Berardi U. Destructive interferences created using additive manufacturing. 2017;45.

[38] Cai X, Guo Q, Hu G, Yang J. Ultrathin low-frequency sound absorbing panels based on coplanar spiral tubes or coplanar Helmholtz resonators. Appl Phys Lett 2014;105:121901.

[39] Sun B, Kulinsky L. Fabrication of regular polystyrene foam structures with selective laser sintering. Materials Today Communications 2017;13:346-53.

[40] Sauerwein M, Doubrovski EL. Local and recyclable materials for additive manufacturing: 3D printing with mussel shells. Materials Today Communications 2018;15:214-7.

[41] Mrazović N, Baumers M, Hague R, Fischer M. Guiding building professionals in selecting additive manufacturing technologies to produce building components. Materials Today Communications 2018;15:199-202.

[42] Ferro CG, Varetti S, De Pasquale G, Maggiore P. Lattice structured impact absorber with embedded anti-icing system for aircraft wings fabricated with additive SLM process. Materials Today Communications 2018;15:185-9.

[43] Sarakinioti MV, Turrin M, Konstantinou T, Tenpierik M, Knaack U. Developing an integrated 3Dprinted façade with complex geometries for active temperature control. Materials Today

Communications 2018;15:275-9. 
[44] Kumar S, Czekanski A. Roadmap to sustainable plastic additive manufacturing. Materials Today Communications 2018;15:109-13.

[45] Bloomfield M, Borstrock S. Modeclix. The additively manufactured adaptable textile. Materials Today Communications 2018;16:212-6.

[46] Liu Z, Zhan J, Fard M, Davy JL. Acoustic properties of a porous polycarbonate material produced by additive manufacturing. Materials Letters 2016;181:296-9.

[47] Gao N, Hou H. Sound absorption characteristic of micro-helix metamaterial by 3D printing. Theoretical and Applied Mechanics Letters 2018;8:63-7.

[48] Dupont T, Leclaire P, Panneton R, Umnova O. A microstructure material design for low frequency sound absorption. Applied Acoustics 2018;136:86-93.

[49] Pinho PG, Pinto M, Almeida RMSF, Lemos LT, Lopes SM. Aspects concerning the acoustical performance of school cafeterias. Applied Acoustics 2018;136:36-40.

[50] Park JH, Sohn CH. On optimal design of half-wave resonators for acoustic damping in an enclosure. Journal of Sound and Vibration 2009;319:807-21.

[51] Cai C, Mak CM. Noise control zone for a periodic ducted Helmholtz resonator system. J Acoust Soc Am 2016;140:EL471-7.

[52] Yongnian Y, Shengjie L, Renji Z, Feng L, Rendong W, Qingping L et al. Rapid Prototyping and Manufacturing Technology: Principle, Representative Technics, Applications, and Development Trends. Tsinghua Science \& Technology 2009.

[53] Schmid M, Kleijnen R, Vetterli M, Wegener K. Influence of the origin of polyamide 12 powder on the laser sintering process and laser sintered parts. Appl Sci 2017;7.

[54] BSEN ISO 10534-2: 2001. Determination of sound absorption coefficient and impedance in impedance tubes, Part 2: Transfer-function method. 2001.

[55] BS EN ISO 10140-1. Acoustics. Laboratory measurement of sound insulation of building elements. Application rules for specific products. 2016.

[56] Han J, Herrin DW, Seybert AF. Accurate Measurement of Small Absorption Coefficients. SAE International 2007:1-5.

[57] Galindo J, Serrano JR, Arnau FJ, Piqueras P. High-frequency response of a calculation methodology for gas dynamics based on Independent Time Discretisation. Mathematical and Computer Modelling 2009;50:812-22. 